\title{
Head Impact Exposure and Biomechanics in University Varsity Women's Soccer
}

\author{
Rebecca Kenny, ${ }^{1}$ Marko Elez, ${ }^{2}$ Adam Clansey, ${ }^{3}$ Naznin Virji-Babul, ${ }^{4}$ \\ and Lyndia C. Wu (i) ${ }^{3}$ \\ ${ }^{1}$ Department of Rehabilitation Sciences, The University of British Columbia, 2177 Wesbrook Mall, Vancouver, BC V6T 1Z3, \\ Canada; ${ }^{2}$ Department of Integrated Sciences, The University of British Columbia, 6356 Agricultural Rd Room 464, Vancouver, \\ BC V6T 1Z2, Canada; ${ }^{3}$ Department of Mechanical Engineering, The University of British Columbia, 6250 Applied Science Ln \\ \#2054, Vancouver, BC V6T 1Z4, Canada; and ${ }^{4}$ Department of Physical Therapy, The University of British Columbia, 2177 \\ Wesbrook Mall, Vancouver, BC V6T 1Z3, Canada
}

(Received 1 September 2021; accepted 1 January 2022; published online 18 January 2022)

Associate Editor Stefan M Duma oversaw the review of this article.

\begin{abstract}
Soccer is a unique sport where players purposefully and voluntarily use their unprotected heads to manipulate the direction of the ball. There are limited soccer head impact exposure data to further study brain injury risks. The objective of the current study was to combine validated mouthpiece sensors with comprehensive video analysis methods to characterize head impact exposure and biomechanics in university varsity women's soccer. Thirteen female soccer athletes were instrumented with mouthpiece sensors to record on-field head impacts during practices, scrimmages, and games. Multi-angle video was obtained and reviewed for all on-field activity to verify mouthpiece impacts and identify contact scenarios. We recorded 1307 video-identified intentional heading impacts and 1011 video-verified sensor impacts. On average, athletes experienced 1.83 impacts per athlete-exposure, with higher exposure in practices than games/scrimmages. Median and 95th percentile peak linear and peak angular accelerations were 10.0, $22.2 \mathrm{~g}$, and 765, $2296 \mathrm{rad} / \mathrm{s}^{2}$, respectively. Long kicks, top of the head impacts and jumping headers resulted in the highest impact kinematics. Our results demonstrate the importance of investigating and monitoring head impact exposure during soccer practices, as well as the opportunity to limit high-kinematics impact exposure through heading technique training and reducing certain contact scenarios.
\end{abstract}

Keywords-Head impact exposure, Repetitive head impacts, Soccer heading, Female athletes, Mouthpiece sensor, Video verification, Head impact kinematics.

Address correspondence to Lyndia C. Wu, Department of Mechanical Engineering, The University of British Columbia, 6250 Applied Science Ln \#2054, Vancouver, BC V6T 1Z4, Canada. Electronic mail: 1wu@mech.ubc.ca

\section{INTRODUCTION}

Soccer is one of the most watched and most played sports in the world, with more than 265 million people playing soccer worldwide, and upwards of 22 million youth involved in the game. ${ }^{23}$ The FIFA World Cup 2018 in Russia reached over three and a half billion viewers, more than half the global population over the age of four, which is indicative of the popularity of the game worldwide. ${ }^{10}$ Soccer is also a unique sport where players purposefully and voluntarily use their unprotected heads to manipulate the direction of the soccer ball for both offensive and defensive plays. ${ }^{21}$ As such, heading the ball is an integral part of the game. On average, players head the ball 6 to 12 times per game, with ball velocities reaching up to $80 \mathrm{~km} / \mathrm{h} .{ }^{48}$ However, such high ball velocities are rare and the most common headers occur at velocities lower than $65 \mathrm{~km} / \mathrm{h} .{ }^{26}$ Aside from game scenarios, soccer practices can involve repetitive, low-velocity heading with a focus on skill development. In recent years, there is increasing concern of soccer heading effects on the brain, prompting rule changes such as limiting heading exposure in children. ${ }^{46,51}$ However, there are limited data to quantify the exposure level and biomechanics of soccer head impacts to further study brain injury risks.

The ball-to-head impact during heading generates impulsive head accelerations, thought to be correlated with concussions or mild traumatic brain injury (mTBI) risk. ${ }^{32}$ Angular head acceleration has long 
been hypothesized to correlate with closed-head mTBI risk. ${ }^{18,}$ 30, 37 Specifically, Holbourn proposed that brain deformation occurs with angular head acceleration due to the low shear modulus of the brain. ${ }^{18}$ Based on primate studies, angular head accelerations indeed produce diffuse deformations across the brain, while linear head accelerations can lead to focal trauma. ${ }^{37}$ Given the paucity of human data, recent studies have been focused on measuring head impacts with full 6 degrees-of-freedom (6DOF) kinematics to test previous injury mechanism hypotheses in humans. In simulated heading drills with the ball traveling between 32 and $43 \mathrm{~km} / \mathrm{h}$, head linear acceleration was measured to be between 15 and $20 \mathrm{~g}$ and angular acceleration between 1000 and $2000 \mathrm{rad} / \mathrm{s}^{2} .{ }^{35}$ With recent development of wearable head impact sensors, studies have also begun to quantify on-field sports head impact exposure and kinematics.

Wearable head impact sensors have various form factors, ranging from helmet sensors ${ }^{8,42,43}$ and headgear sensors, ${ }^{5}$ to headbands, ${ }^{13}$ skin patches,${ }^{36}$ and mouthguards. 3, 7, 47, 54 Helmet and headgear sensors can be applied to helmeted sports (e.g., football, hockey) or headgear sports (e.g., boxing), but do not work with non-helmeted sports such as soccer. In addition, these sensors may not accurately measure head kinematics due to helmet or headgear dislocation. ${ }^{19}$ Skin patch measurements have been shown to be affected by skin movement artifacts, ${ }^{56}$ leading to potential overestimation of both the number and kinematics of head impacts in field studies. ${ }^{40}$ Mouthguard-based sensors offer a more direct measurement of skull acceleration by mounting the sensor on upper dentition. ${ }^{17}$ Custommade mouthguards have a similar form factor as regular athletics mouthguards, and have been deployed in American football, Australian rugby union, and boxing. ${ }^{2}, 12,16,20,28$ More recently, custom-fitted mouthpieces with sensors embedded in acrylic and molded to the posterior side of the upper dentition have also been validated for head impact measurement, where peak linear acceleration, angular velocity, and angular acceleration measurements linearly correlate with reference measurements with $r^{2} \geq 0.95$ and slope falling within $1 \pm 0.04 .^{41}$ Additionally, through video verification of on-field head impacts, the mouthpieces showed an overall sensitivity of $69.2 \%$ and a positive predictive value of $80.3 \%$ in head impact detection. Such mouthpiece sensors are easily adopted in sports such as soccer, where athletes do not typically wear mouthguards, and have been piloted in U13-U14 girls soccer, ${ }^{33,41,49}$ and collegiate women's soccer. ${ }^{11}$

Previous research seeking to establish head impact exposure in soccer have mostly relied on subjective self reporting. ${ }^{27,}{ }^{53}$ Self reporting was found to be unreliable in youth girls soccer, with players overestimating the number of headers experienced over the course of a season. ${ }^{15}$ As an alternative, video analysis provides a more objective measurement tool for head impact exposure. However, video analysis is time consuming and past research has attempted to mitigate the time commitment by analyzing a few practices and/or games ${ }^{22}$ or have only intermittently collected data ${ }^{44}$ to estimate an average number of headers. This selective method may result in a sampling bias, and a more comprehensive sample may be necessary to quantify head impact exposure more accurately in soccer. In addition, due to inaccuracies in on-field head impact detection by impact sensors, video-verification of head impacts are still necessary for validating sensor-measured exposures. ${ }^{39} \mathrm{~A}$ recent systematic review evaluated 19 soccer head impact exposure studies to collect impact kinematics data, ${ }^{38}$ and 8 of these studies did not perform video or observer confirmation for all sensor-recorded events.

A systematic review conducted by McCunn et al. examined twelve studies that conducted on-field research with youth and collegiate soccer players. ${ }^{31}$ Most studies focused on games as the optimal setting to record heading activity, leaving a paucity of practice data even though practice usually represent a larger percentage of heading exposure as players age. ${ }^{4}$ Additionally, 8 of the 12 field studies focused on youth athletes, indicating a need to further investigate header biomechanics in older players, including university/collegiate athletes. The reporting of exposure and head impact kinematics have included inconsistent metrics, making it difficult to compare and aggregate information from different studies. An average of one to nine headers per player per game was reported, though there was a variety of ways to report, including per athlete exposure, per hour of play or just an overall number of headers observed. When investigating the biomechanics of heading, linear acceleration was the most common outcome variable present in almost all studies evaluated, while angular velocity was reported in just over half of the studies and angular acceleration was reported in only 4 of the 12 field studies ( 7 of the 36 studies). Most studies applied skin patch and headband sensors, and the average peak linear acceleration and angular acceleration varied greatly, ranging from 4 to $50 \mathrm{~g}$ and negligible to $4500 \mathrm{rad} / \mathrm{s}^{2}$, respectively. Since the publication of the systematic review, only one study has applied mouthpiece sensors to collegiate women's soccer. ${ }^{11}$ Based on the limitations presented by the recent systematic review, soccer head impact exposure studies need to include both practices and games, include older athletes, and capture both linear and angular head kinematics using validated sensors.

The objective of the current study was to combine validated $6 \mathrm{DOF}$ mouthpiece sensors ${ }^{34}$ with indepen- 
dent video analysis methods to quantify head impact exposure and biomechanics in university varsity women's soccer. In addition, we conducted a comprehensive analysis of exposure levels and head impact kinematics across session type, player position, and contact scenarios to identify key factors affecting impact exposure and severity. Our goal in this research is to expand the current limited dataset of sports head impact exposure and biomechanics to inform potential acute and cumulative injury risks.

\section{METHODS}

Thirteen female university varsity athletes, including 6 defenders, 4 midfielders, and 3 forwards, were recruited from a university varsity soccer team (age: $19.9 \pm 1.6$, height: $169.3 \pm 6.3 \mathrm{~cm}$, weight: $65.5 \pm 5.2 \mathrm{~kg}$ ). Participants were required to be active participants during the season and able to wear an instrumented mouthpiece sensor, designed by Wake Forest Center for Injury Biomechanics. ${ }^{41}$ Dental impressions of the athletes were obtained by a trained dental professional. The dental impressions were then used to create a dental mold. A custom-fitted mouthpiece, made of acrylic material, was constructed for each athlete. The study protocol was approved by the University of British Columbia Ethics Board (UBC REB H17-02973) and all players provided informed consent to participate.

Data were collected during the 2020-2021 seasons, including both the fall and spring seasons. Figure 1 illustrates our video and mouthpiece data collection methodology. On-field data were collected during 77 practices and 57 scrimmages, 12 inter-squad games and 3 competitive games. Due to the COVID-19 pandemic, games were cancelled starting in October 2020, resulting in only three games being played during the season. Even though only 3 official games were played, there were 12 inter-squad games and 57 scrimmages (i.e., 7v7, 8v8, 9v9). For practices that involved both practice drills and scrimmages, time on field was divided between the two types and data was separated based on when the head impact occurred (either during a drill or during a scrimmage). All athletes were handed their custom mouthpieces before each practice and game. Players had the option to decline wearing the mouthpiece before each practice or game or to remove their mouthpiece at any point during each practice or game.

Each mouthpiece contained a tri-axis accelerometer, to measure translational acceleration in the anterior/posterior $(X)$, left/right $(Y)$ and superior/inferior $(Z)$ directions, and a tri-axis gyroscope, to measure angular velocity in the coronal $(X)$, sagittal $(Y)$, and horizontal $(Z)$ planes (Fig. 1c). The mouthpieces were set to trigger impact recording when the sensor detected over $5 \mathrm{~g}$ linear acceleration for at least $2 \mathrm{~ms}$ in any of the $X, Y$ or $Z$ axes. A low trigger threshold of $5 \mathrm{~g}$ linear acceleration was selected based on pilot testing and previous research that indicated $10 \mathrm{~g}$ linear acceleration was not sensitive enough to detect mild soccer headers. ${ }^{13}, 33,41$ This threshold also matches research conducted recently using the same mouthpiece sensors. ${ }^{49}$ Once the mouthpiece was triggered, head kinematics data were collected at $1000 \mathrm{~Hz}$ for $150 \mathrm{~ms}$ (30 ms pre-impact, $120 \mathrm{~ms}$ post-impact). After each practice or game, impact data were downloaded from each mouthpiece.

Multi-angle time-synchronized video, with $2.7 \mathrm{~K}$ resolution at 60 frames-per-second, was collected during each on-field activity, including both games and practices (Figs. 1a and 1b). A second-resolution clock synched with the mouthpieces was shown to each video camera at the beginning and end of any on-field activity. All videos were reviewed independently of mouthpiece sensor recordings to identify the timing of header events and evaluate the contact scenario. The lead author RK conducted a second round of video review to confirm each impact. In this study, the focus was on intentional ball-to-head impacts, since unintentional head impacts, such as head-to-ground or head-to-head, were not observed.

Each ball-to-head impact was categorized based on session type (games and scrimmages, practices), player position (forward, midfield, defender), and contact scenario (based on ball delivery method, impact location, and player stance). We grouped scrimmages with games instead of practices since activity during scrimmages were more similar to game activity. Figure 1d shows a detailed breakdown of the contact scenarios. The ball delivery method, based on the last contact the ball had before impacting the player's head, was categorized into long kick $(>10 \mathrm{~m})$, short kick $(<10 \mathrm{~m})$, overhand throw, underhand throw, player (the ball hits another player before impacting the head) and ground (ball hits the ground before contact with the player). The impact location, based on where the ball hits the player's head, was categorized into forehead, top, side (left/right), and back. The player stance, based on how the player was positioned before ball contact, was categorized into jumping or non-jumping (indicating a player had one or both feet contacting the ball during head contact). Other contact scenarios, such as falls or player-to-player collisions, were not observed and not considered.

Once video analysis was completed, we quantified head impact exposure levels using video-confirmed headers. Head impact exposure was defined both in terms of head impacts per athlete exposure or head 
(a)

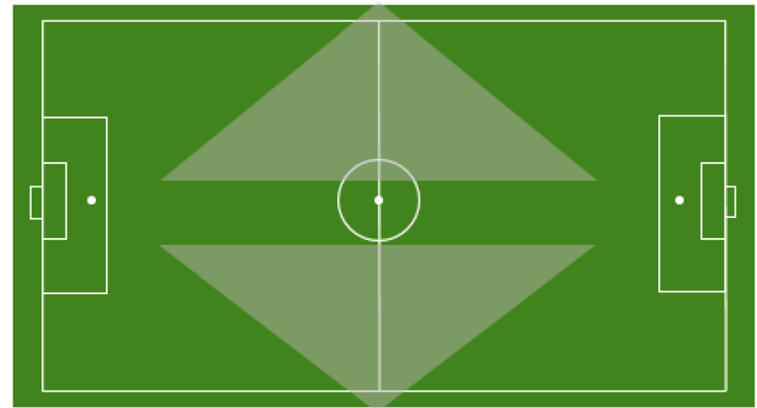

(d)

View 2

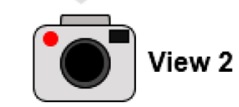

(b)

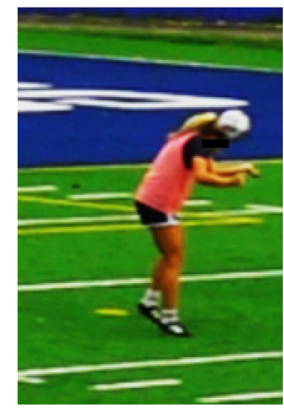

View 1
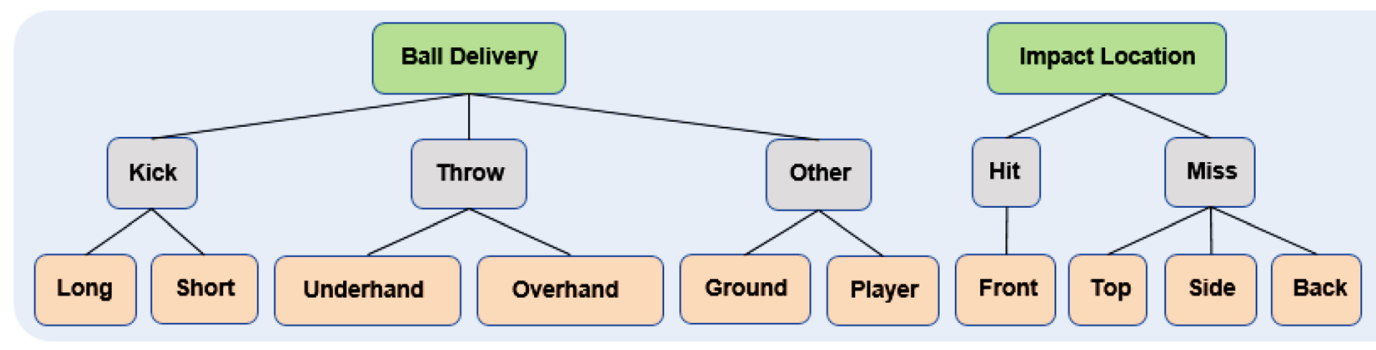

(c)

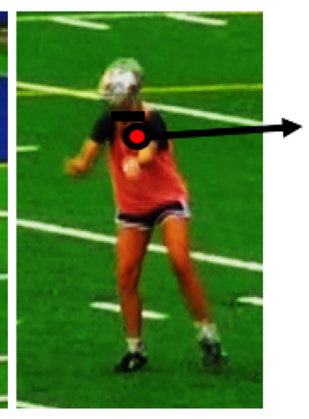

View 2

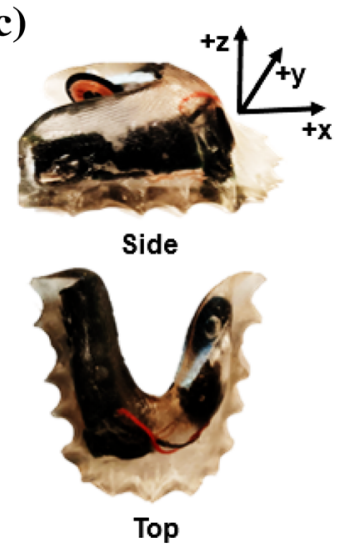

FIGURE 1. Methodology. For each practice or game, at least two camera views were recorded (a), such that any header can be clearly confirmed in at least one view (b). The athletes were wearing instrumented mouthpieces (c) that recorded full 6-degree-offreedom linear and rotational head kinematics. Panel (D) summarizes video analysis classifications used during video analysis to categorize each intentional head impact into a contact scenario. The 13 contact scenarios (in orange, further dividing side impacts into left/right impacts) are organized by the overall categories in green.

impacts per hour of play. An athlete exposure (AE) is defined as one instrumented athlete per session (single practice, scrimmage, or game). The impacts per AE and impacts per hour are calculated by dividing the total number of impacts over the total number of $\mathrm{AE}$ and total hours of on-field play, respectively. For head impact kinematics analysis, each video-verified head impact was time-synchronized and compared with mouthpiece data collected from each athlete. The impact kinematics data were transformed from the device coordinate system to the head's coordinate system at head center-of-gravity. ${ }^{41}$ After transformation, a lowpass Butterworth filter at 300 and $258 \mathrm{~Hz}$ cutoff was used for accelerometer and gyroscope data, respectively. ${ }^{9}{ }^{16,50}$ Peak values of resultant linear acceleration, angular velocity and acceleration were calculated for each video-verified mouthpiece event. In addition to peak angular velocity, we also calculated the peak change in angular velocity for each impact, since soccer heading impacts mostly have non-zero initial angular velocities. To characterize the peak kinematics distributions, we quantified the median and 95th percentile peak values, and organized these across session type, player position, and contact scenario. We ran a Shapiro-Wilks normality test to find that the data were not normally distributed. As such we ran nonparametric statistical comparisons with the Kruskal-Wallis test using MATLAB's kruskalwallis function was performed to compare the mean peak kinematics across the different impact categories. Then, a pairwise comparison of the group means was performed using the MATLAB multcompare function. MATLAB R2020a (Mathworks, Natick, USA) was used for data processing and statistical analyses.

\section{RESULTS}

From video analysis, we identified 1307 confirmed headers across 13 participants wearing mouthpieces over the course of 77 practices, 57 scrimmages, 12 inter-squad games, and 3 competitive games. The majority of head impacts were identified during practices $(n=1200)$, and fewer were identified from scrimmage and games $(n=107)$. The total number of athlete exposures on field was 609, and the total number of hours of play across all participants was $722.93 \mathrm{~h}$, with a breakdown shown in Table 1 . We summarized the head impact exposure per hour and per athlete exposure in Table 2, comparing session

\section{BMES


TABLE 1. Hours of play and athlete-exposures by player position.

\begin{tabular}{lrl}
\hline Activity & Hour of play $(\mathrm{h})$ & Athlete-exposure $(\mathrm{AE})$ \\
\hline Practice & 518.82 & 402 \\
Forwards & 84.32 & 61 \\
Midfielders & 171.05 & 133 \\
Defenders & 325.82 & 208 \\
Games/scrimmages & 204.11 & 313 \\
Forwards & 26.95 & 41 \\
Midfielders & 73.35 & 109 \\
Defenders & 103.82 & 163 \\
Total & 722.93 & 715 \\
\hline
\end{tabular}

type, player position, and contact scenario. On average, athletes experienced $1.81 \mathrm{impacts} / \mathrm{h}$ (game/scrimmage: 0.52 , practice: 2.31 ) and 1.83 impacts/AE (game/ scrimmage: 0.34, practice: 2.99). Comparing across contact scenarios, the most common ball delivery method was underhand throw, followed by player-toplayer. The most common impact location was forehead, followed by top of the head impacts. In addition, players were more likely to be planted while heading than jumping.

Of the 1307 head impacts confirmed via video, we could identify time-synchronized mouthpiece recordings for 1055 impacts. 206 video-confirmed impacts $(15.8 \%)$ did not register on the mouthpiece sensors, indicating these impacts were likely below the $5 \mathrm{~g}$ threshold. For these missed impacts, we verified that the sensors were functioning properly, recorded other impacts during the same activity sessions, and passed a quality check after each session. 44 of the 1055 mouthpiece impacts were removed due to gyroscope sensor error. A total of 1011 impacts were used for head impact kinematics analysis. Figure 2 shows representative impact kinematic plots, including example long kicks (Fig. 2a and 2b) that led to relatively high impact kinematics and underhand/overhand throw impacts that involved relatively low impact kinematics (Figs. 2c and 2d), across different impact locations and player stance conditions. Front (forehead) impacts typically resulted in linear accelerations in both the negative $X$ (posterior) and negative $Z$ (downwards) directions with sagittal angular accelerations (Fig. 2a). Top impacts could result in higher linear accelerations in the downwards direction (Fig. 2b). Some impacts (Figs. 2a and 2d) involved higher initial head angular velocities prior to the impact. Overall, we report median peak linear acceleration of $10.0 \mathrm{~g}$, peak angular velocity of $5.4 \mathrm{rad} / \mathrm{s}$, peak change in angular velocity of $4.2 \mathrm{rad} / \mathrm{s}$, and peak angular acceleration of $765 \mathrm{rad} / \mathrm{s}^{2}, 95$ th percentile peak linear acceleration of $22.2 \mathrm{~g}$, peak angular velocity of $11.3 \mathrm{rad} / \mathrm{s}$, peak change in angular velocity of $8.8 \mathrm{rad} / \mathrm{s}$, and peak angular acceleration of $2296 \mathrm{rad} / \mathrm{s}^{2}$. Box scatterplots and summary statistics of the peak resultant head kinematics are shown in Fig. 3 (session type and player position), Fig. 4 (contact scenarios), and Table 3 (all summary statistics).

We ran Kruskal-Wallis tests and pairwise comparisons to compare peak impact kinematics distributions across each impact category. When comparing session types, games were significantly higher than practices in mean peak linear acceleration, angular velocity, and angular acceleration $(p<0.001)$. When comparing ball delivery methods, mean peak linear and angular acceleration in long kicks were significantly higher than short kicks, underhand throw, overhand throws, player-to-player, and ground-to-player impacts $(p<0.001)$. Long kicks were not significantly different from overhand throws in angular velocity $(p<0.5)$ but were significantly higher for all other comparisons in angular velocity $(p<0.001)$. When comparing impact location, top of the head impacts were significantly higher than forehead impacts in all mean peak kinematics $(p<0.001)$. When comparing player stance, jumping head impacts were significantly higher than non jumping head impacts in mean peak kinematics $(p<0.001)$. When comparing player position, mean peak kinematics by defenders were significantly higher than midfielders and forwards $(p<0.001)$ but there were no significant differences between midfielders and forwards.

\section{DISCUSSION}

The objective of the current study was to evaluate on-field head impact exposure in university varsity women's soccer players and analyze head impact kinematics measured using a mouthpiece sensor. We provide comprehensive analyses of exposure levels and head impact kinematics across different player positions during contact scenarios experienced in both practices and games. The results of this study show that it is important to monitor practice head impact exposure due to potentially comparable or higher impact count during practices, as well as the incidence of high-kinematics impacts during practices. In addition, we demonstrate that certain contact scenarios may be associated with higher kinematics head impacts, which may lead to implications in soccer training and heading technique.

We report a higher average number of impacts per athlete exposure compared to previous studies, ${ }^{25,}, 45$ 
TABLE 2. Average head impact exposures by session type, player position, and contact scenario.

\begin{tabular}{|c|c|c|c|c|}
\hline & Game/scrim.Impacts/h & Game/scrim.Impacts/AE & Practicelmpacts $/ \mathrm{h}$ & Practicelmpacts/AE \\
\hline Average & 0.52 & 0.34 & 2.31 & 2.99 \\
\hline \multicolumn{5}{|l|}{ Player position } \\
\hline Defender & 0.57 & 0.36 & 1.86 & 2.91 \\
\hline Midfield & 0.50 & 0.34 & 1.73 & 2.23 \\
\hline Forward & 0.41 & 0.27 & 3.55 & 4.90 \\
\hline \multicolumn{5}{|l|}{ Ball delivery } \\
\hline Long kick & 0.29 & 0.19 & 0.21 & 0.27 \\
\hline Short kick & 0.09 & 0.06 & 0.16 & 0.21 \\
\hline Overhand throw & 0.00 & 0.00 & 0.05 & 0.06 \\
\hline Underhand throw & 0.00 & 0.00 & 0.95 & 1.22 \\
\hline Player & 0.06 & 0.04 & 0.91 & 1.16 \\
\hline Ground & 0.08 & 0.05 & 0.03 & 0.04 \\
\hline \multicolumn{5}{|l|}{ Impact location } \\
\hline Forehead & 0.23 & 0.15 & 1.33 & 1.70 \\
\hline Top & 0.28 & 0.19 & 0.94 & 1.20 \\
\hline Left & 0.00 & 0.00 & 0.02 & 0.02 \\
\hline Right & 0.00 & 0.00 & 0.02 & 0.02 \\
\hline Back & 0.00 & 0.00 & 0.00 & 0.00 \\
\hline \multicolumn{5}{|l|}{ Player stance } \\
\hline Jump & 0.29 & 0.19 & 0.35 & 0.44 \\
\hline No jump & 0.23 & 0.15 & 1.96 & 2.50 \\
\hline
\end{tabular}

(a) Long Kick, Front Jump
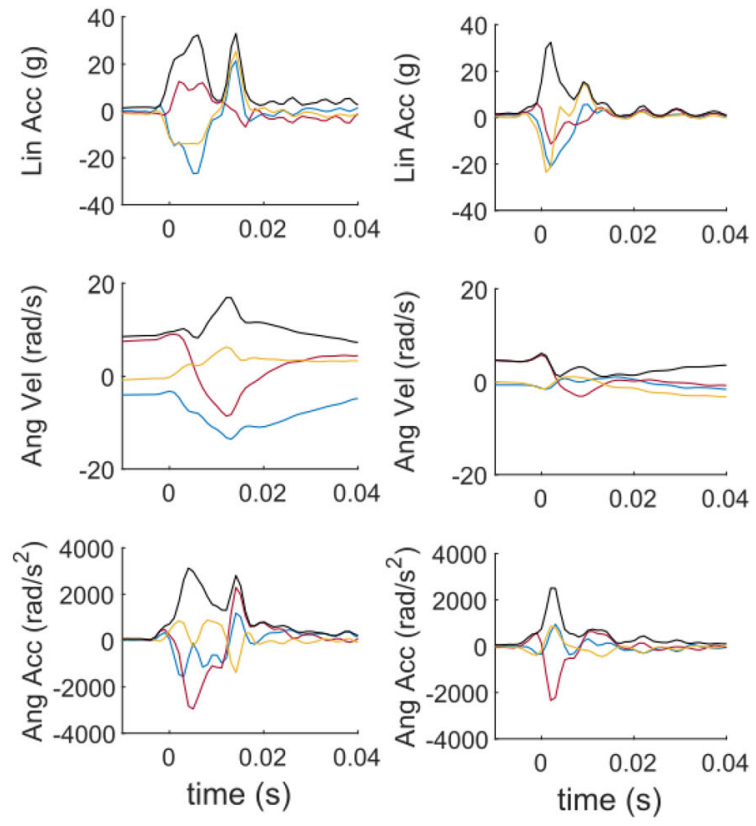

(b) Long Kick, Top, Jump
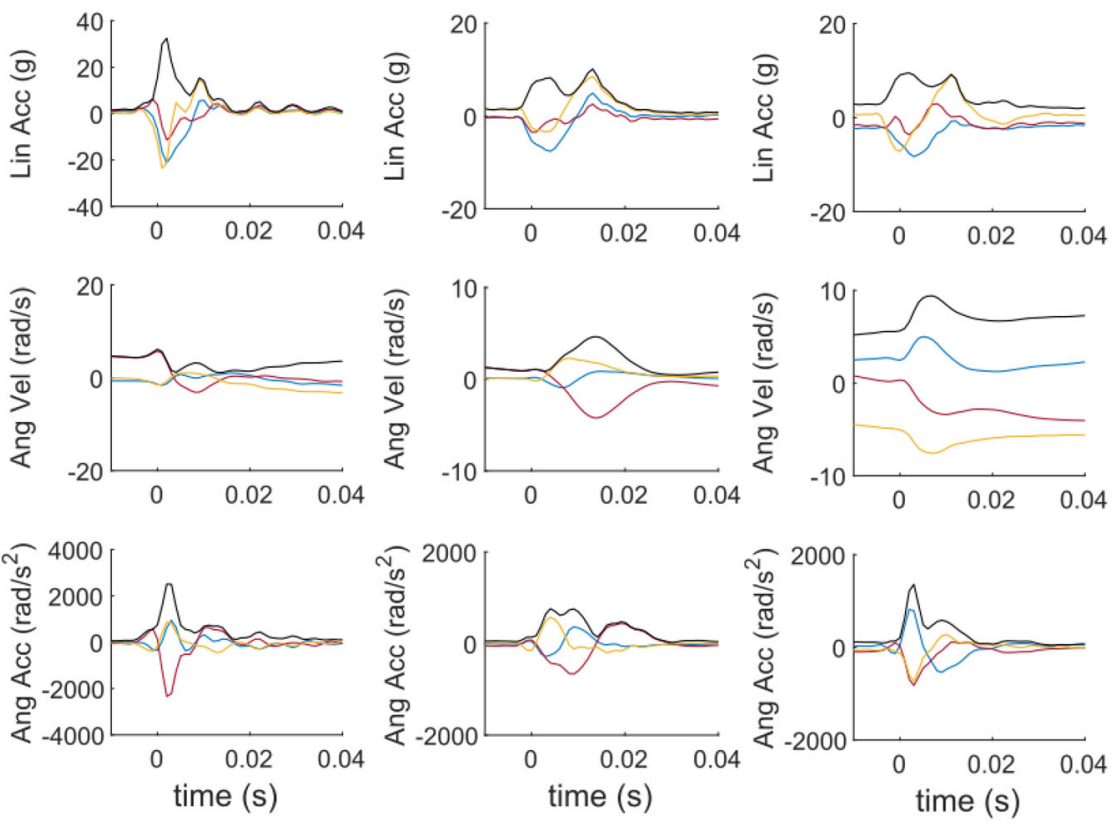

FIGURE 2. Representative head impact kinematics plots. Here we compare example long kick headers with observed impact locations at the front (a) and top of the head (b), a low-kinematics underhand throw header (c), and an overhand throw header that exhibited high initial angular velocity (d). 
(a)

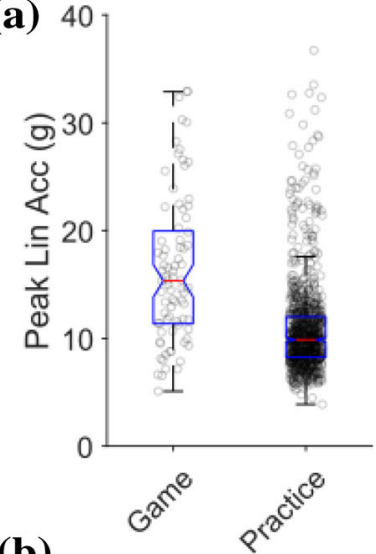

(b)

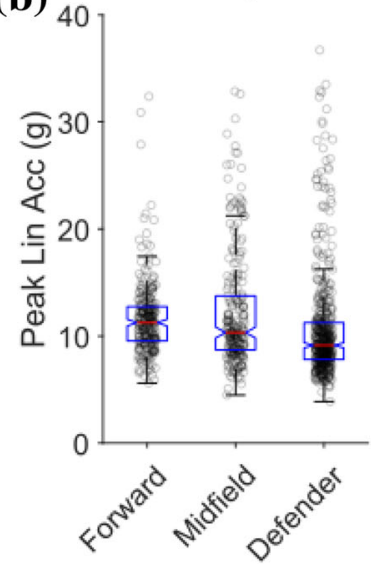

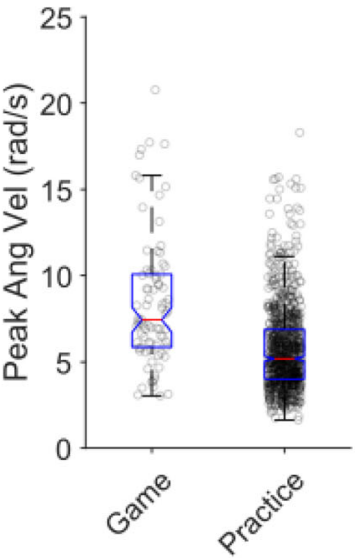

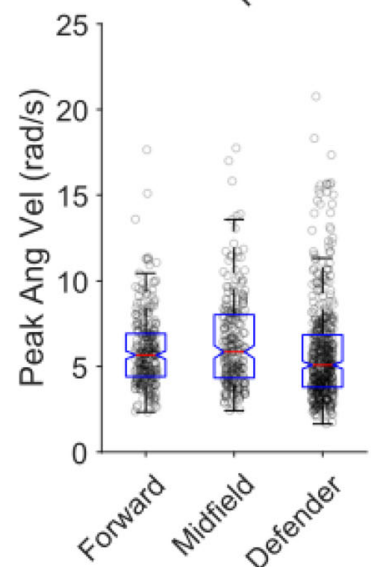

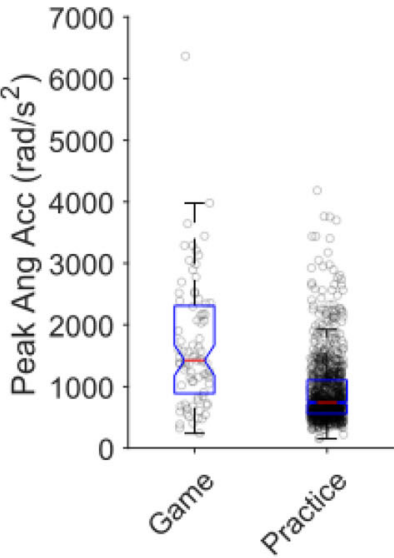

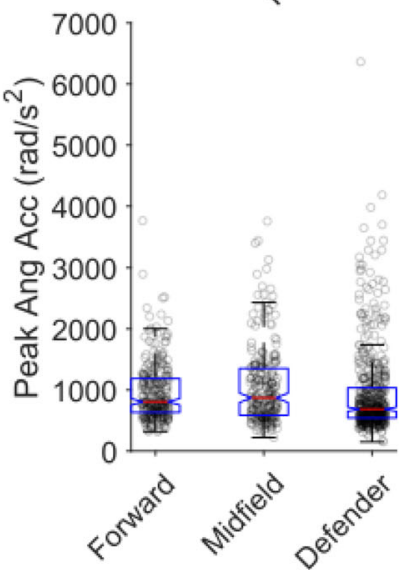

FIGURE 3. Box scatter plots of peak head impact kinematics across session type and player position. Here we compare the distributions of peak impact kinematics across (a) session type, and (b) player position. Individual circles represent each impact and the box plot show the 75th percentile and 25th percentile peaks with the red line representing the median.

which is likely attributed to the unique practice-focused season during the COVID-19 pandemic. Lamond et al. $^{25}$ and Saunders et al. ${ }^{45}$ both reported 0.67 heading impacts/AE in NCAA collegiate women's soccer athletes, compared to 1.83 impacts/AE in the current paper. During the 2020-2021 season, there were few games and more emphasis on skill development. This resulted in a high number of heading drills and focus on heading technique training, providing us with a unique data set. Correspondingly, the practice heading exposure in the current study (2.99 impacts/ $\mathrm{AE})$ is substantially higher than those reported in prior studies (0.65 impacts/AE in Saunders et al. ${ }^{45}$ and 0.03 impacts/AE in Lamond et $a .^{25}$ ), while the game heading exposure of 0.34 impacts/AE falls within the range of previously reported exposures (0.17 impacts/ $\mathrm{AE}^{25}$ and 0.71 impacts $/ \mathrm{AE}^{45}$ ). The wide variance in exposure level reported for practices can also be attributed to the training and playing style for different teams. While past studies have mainly been focused on quantifying head impact exposure during games, we show that exposure in practices can be comparable or even higher than that during games. As such, head impact exposure during practices should also be quantified to understand total head impact exposure in soccer athletes.

We evaluated the effect of player position on head impact exposure in both practices and games. While practices are not always position specific and previous research has mostly focused on evaluating position effects during games, there were times during practices that players broke into position-based drills (such as practicing free kicks, corner kicks, crossing drills). Forwards had higher impact exposures during practices, while defenders had higher exposure in scrimmages and games as well as higher overall exposure. Lamond et al. also reported higher exposure in defenders (1.22 impacts/AE) compared with midfielders (0.85 impacts/AE) and forwards (0.55 impacts/ $\mathrm{AE}){ }^{45}$ Two other studies reported higher exposure in midfielders, followed by defenders and forwards. ${ }^{29,} 40$ Our exposure results are slightly higher Lamond et $a l^{45}$ and lower than Lynall et al., ${ }^{29}$ who both studied NCAA Division I female soccer players. Of note, in the current study, we had more defenders $(n=6)$ than midfielders $(n=4)$ and forwards $(n=3)$. Future 

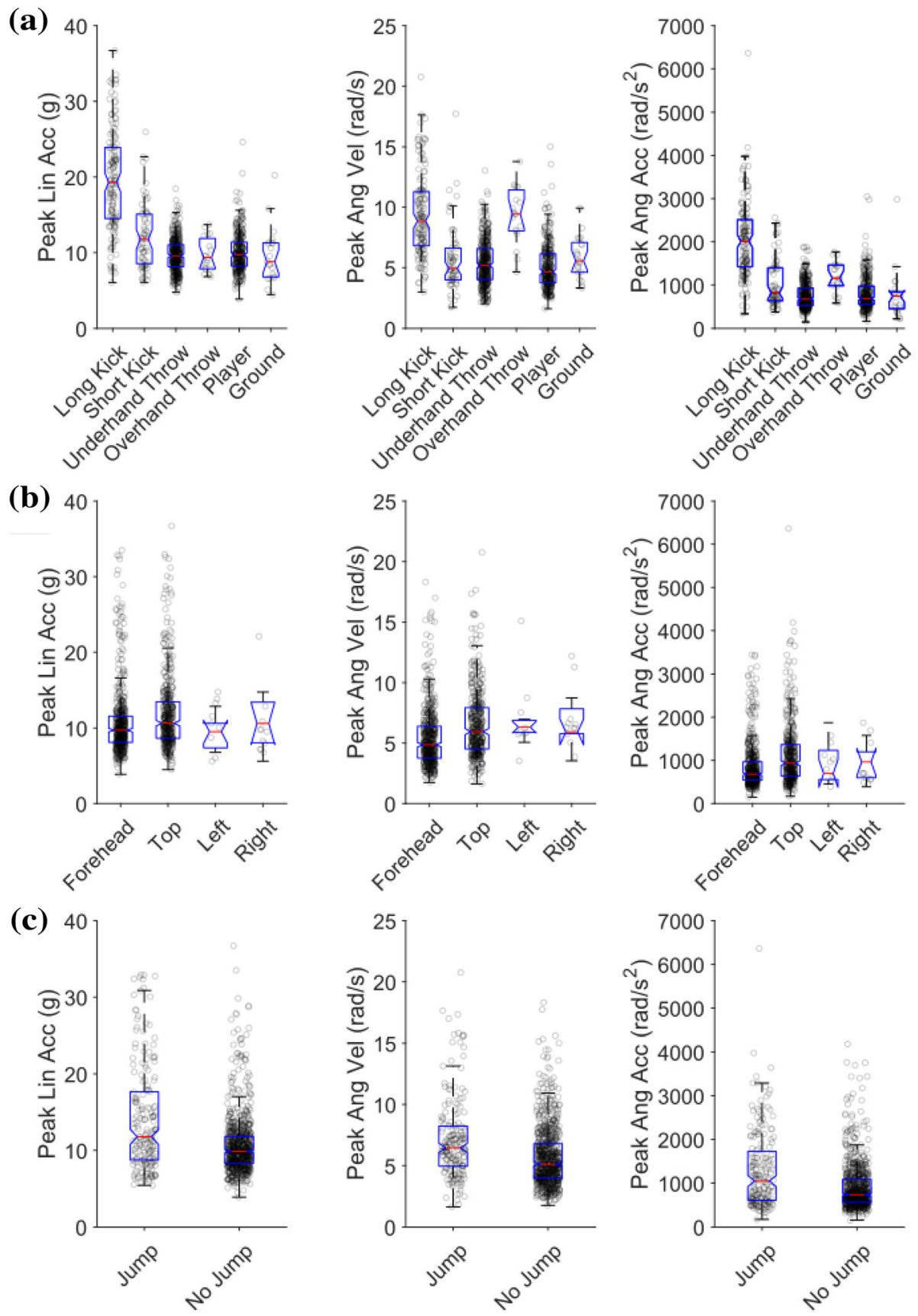

FIGURE 4. Box scatter plots of peak head impact kinematics across contact scenarios. Here we compare the distributions of peak impact kinematics across (a) ball delivery methods, (b) impact locations and (c) player stance. Individual circles represent each impact and the box plot show the 75th percentile and 25th percentile peaks with the red line representing the median.

research should continue to explore the number of head impacts per position as there are a handful of player specific drills that take place during practice and these drills could influence overall exposure numbers.

Our head impact kinematics results measured by mouthpiece sensors are generally lower than those reported in previous studies using skin patches and headband sensors in collegiate women's soccer players. Caccese et al. used a headband sensor and reported average peak linear and angular acceleration of $28.2 \mathrm{~g}$ and $7100 \mathrm{rad} / \mathrm{s}^{2}, 6$ compared with $11.3 \mathrm{~g}$ and $976 \mathrm{rad} / \mathrm{s}^{2}$ in the current study. Lynall et al. used a skin patch sensor and reported higher median and 90th percentile angular accelerations of 2138.39 and $4430.04 \mathrm{rad} / \mathrm{s}^{2}$, which are substantially higher than the values found in the current paper while the peak linear accelerations are comparable. Also using skin patch sensors, Press and Rowson reported high peak kinematics in some 
TABLE 3. Summary of impact kinematics across session type, player position, and contact scenario.

\begin{tabular}{|c|c|c|c|c|c|c|c|c|c|c|c|c|c|}
\hline & \multirow[b]{2}{*}{$n$} & \multicolumn{3}{|c|}{ Peak lin accel (g) } & \multicolumn{3}{|c|}{ Peak ang vel (rad/s) } & \multicolumn{3}{|c|}{ Peak $\Delta$ ang vel (rad/s) } & \multicolumn{3}{|c|}{ Peak ang accel $\left(\mathrm{rad} / \mathrm{s}^{2}\right)$} \\
\hline & & Mean & Median & 95th pc & Mean & Median & 95th pc & Mean & Median & 95th pc & Mean & Median & 95th pc \\
\hline $\begin{array}{l}\text { Total } \\
\text { Activity type }\end{array}$ & 1011 & 11.3 & 10.0 & 22.2 & 6.0 & 5.4 & 11.3 & 4.6 & 4.2 & 8.8 & 976 & 765 & 2296 \\
\hline Game/Scr. & 81 & 16.3 & 15.3 & 30.2 & 8.5 & 7.4 & 17.2 & 6.6 & 6.1 & 13.3 & 1669 & 1427 & 3358 \\
\hline $\begin{array}{l}\text { Practice } \\
\text { Player position }\end{array}$ & 930 & 10.9 & 9.9 & 20.8 & 5.7 & 5.2 & 10.6 & 4.4 & 4.1 & 7.8 & 916 & 740 & 2117 \\
\hline Defense & 269 & 10.7 & 9.1 & 23.7 & 5.8 & 5.1 & 11.7 & 4.4 & 3.9 & 9.2 & 944 & 684 & 2534 \\
\hline Midfield & 245 & 12.2 & 10.3 & 23.2 & 6.4 & 5.8 & 11.5 & 4.4 & 3.9 & 9.2 & 1059 & 870 & 2409 \\
\hline $\begin{array}{l}\text { Forward } \\
\text { Ball delivery }\end{array}$ & 497 & 11.6 & 11.3 & 17.0 & 5.9 & 5.7 & 9.9 & 4.6 & 4.4 & 7.6 & 961 & 805 & 1877 \\
\hline Long kick & 140 & 19.6 & 19.3 & 32.3 & 9.3 & 8.9 & 15.6 & 7.2 & 6.7 & 12.8 & 2027 & 2012 & 3543 \\
\hline Short kick & 65 & 12.2 & 11.8 & 22.1 & 5.7 & 4.9 & 11.1 & 4.5 & 4.1 & 9.0 & 1058 & 825 & 2262 \\
\hline Underhand Throw & 421 & 9.7 & 9.6 & 13.5 & 5.4 & 5.2 & 8.7 & 4.2 & 4.0 & 6.8 & 756 & 679 & 1308 \\
\hline Overhand Throw & 19 & 9.7 & 9.3 & 13.6 & 9.4 & 9.4 & 12.9 & 5.7 & 5.4 & 9.4 & 1203 & 1159 & 1740 \\
\hline Player & 343 & 10.0 & 9.7 & 14.5 & 5.1 & 4.7 & 8.4 & 4.0 & 3.7 & 6.7 & 803 & 691 & 1484 \\
\hline $\begin{array}{l}\text { Ground } \\
\text { Impact location }\end{array}$ & 21 & 9.6 & 8.8 & 17.8 & 5.9 & 5.6 & 9.0 & 4.4 & 4.2 & 7.6 & 780 & 747 & 2126 \\
\hline Forehead & 550 & 10.8 & 9.7 & 22.2 & 5.4 & 4.9 & 9.9 & 4.3 & 3.9 & 7.8 & 858 & 680 & 2177 \\
\hline Top & 437 & 12.0 & 10.6 & 22.5 & 6.5 & 5.9 & 11.6 & 5.0 & 4.6 & 9.2 & 1125 & 936 & 2543 \\
\hline Left & 11 & 10.2 & 9.5 & 21.6 & 7.0 & 6.3 & 12.1 & 4.3 & 3.7 & 8.9 & 884 & 695 & 1864 \\
\hline Right & 9 & 10.5 & 10.6 & 14.8 & 7.2 & 5.9 & 15.1 & 3.7 & 2.8 & 7.7 & 938 & 964 & 1577 \\
\hline $\begin{array}{l}\text { Back } \\
\text { Player stance }\end{array}$ & 2 & 15.2 & 15.2 & 15.3 & 9.0 & 9.0 & 12.0 & 6.8 & 6.8 & 9.0 & 938 & 1476 & 2194 \\
\hline Jump & 191 & 14.1 & 11.8 & 30.0 & 7.1 & 6.4 & 15.1 & 7.1 & 5.0 & 15.1 & 1291 & 1047 & 2978 \\
\hline No jump & 820 & 10.7 & 9.9 & 18.5 & 5.7 & 5.1 & 10.4 & 4.4 & 4.0 & 7.8 & 1291 & 735 & 1990 \\
\hline
\end{tabular}

impacts, reaching up to $113 \mathrm{~g}$ and $26222 \mathrm{rad} / \mathrm{s}^{2}$, and the authors stated that the high values should be interpreted cautiously due to potential sensor errors. ${ }^{40}$ In comparison with mouthguard sensors, skin patches and headband sensors have shown over-prediction of peak head kinematics of up to a few hundred percent due to skin and headband motion, with higher overprediction of angular kinematics than linear kinematics. ${ }^{55}$ These findings may explain the higher impact kinematics found in prior skin patch and headband sensor studies compared with the current study.

Games and scrimmages resulted in significantly higher mean peak impact kinematics compared with practices, while the upper range of game/scrimmage and practice head impacts were comparable. Few studies have compared game and practice heading kinematics in collegiate soccer. Lamond et al. reported no significant differences between games and practices, but this study did not distinguish between scrimmages and other forms of practice. ${ }^{45}$ Since scrimmage activities are more similar to games than other practice drills, we grouped games and scrimmages together in the current paper to compare with practices. Interestingly, we found that while the majority of practice impacts were distributed in the lower kinematics range (under $15 \mathrm{~g}, 10 \mathrm{rad} / \mathrm{s}$, and $1500 \mathrm{rad} / \mathrm{s}^{2}$ ), the highest kinematics observed during games and practices were comparable (Fig. 3, highest impact in games-32.9 g, $20.8 \mathrm{rad} / \mathrm{s}, 6364 \mathrm{rad} / \mathrm{s}^{2}$, highest impact in practices-36.7 g, $18.3 \mathrm{rad} / \mathrm{s}, 4183 \mathrm{rad} / \mathrm{s}^{2}$ ). This finding further supports the need to track head impact kinematics during practices, since high-severity impacts may also occur during practices despite the relatively lower rate of incidence.

Among different ball delivery methods, long kicks resulted in the highest peak kinematics, which is a consistent finding with prior studies. ${ }^{6,}{ }^{33}$ Filben et al. used the same mouthpiece sensors as the current study and reported the highest head kinematics resulting from goal kicks, followed by corner kicks, and free kicks. ${ }^{11}$ The magnitude of the head kinematics reported by Filben et al. are substantially higher than the long kick ball delivery category in the current study, possibly due to the inclusion of a higher proportion of game data. For impact location, we found the highest 95th percentile linear acceleration and angular acceleration to be from top of the head impacts while the highest 95th percentile for angular velocity was from side impacts. Previous research investigating youth female soccer players by Harriss et al. also reported highest peak linear acceleration and angular velocity for top impacts, followed by side impacts and front impacts. For players stance, higher impact kinematics resulted from jumping head impacts, which is consistent with Tomblin. ${ }^{49} \mathrm{In}$ 
soccer, the proper heading technique is for players to use their forehead. ${ }^{1,21,48}$ Our kinematics results imply that proper heading may lead to lower impact kinematics, while improper technique (top impacts/side impacts) may lead to higher impact kinematics. In addition, limiting long-distance kick and jumping heading may help reduce the exposure to high-kinematics head impacts.

While we focused on university women's soccer players, we examined differences between previous studies evaluating youth and high school female soccer players and our current study. Our reported exposure levels fall within the range reported in prior U13-U16 girls soccer studies. A study investigating U13-U15 girls soccer found 0.07 impacts per athlete exposure in games (74.04 per 1000 match hours). ${ }^{14}$ A study investigating U13 and U16 girls soccer found U13 girls were exposed to 4.6 headers per hour (3.8 headers per hour in games and 5.1 headers per hour in practice) and U16 girls were exposed to 0.7 headers per hour $(0.5$ headers per hour in games and 0.9 headers per hour in practice). ${ }^{49}$ Another study reported 7.2 headers per athlete exposure in U14 girls soccer. ${ }^{41}$ Our peak impact kinematics also fall between the reported values from two previous U13-U16 girls soccer studies. A study investigating U14 girls soccer found the average peak resultant linear and rotational accelerations in games to be $14.8 \mathrm{~g}$ and $1534 \mathrm{rad} / \mathrm{s}^{2}$, respectively. ${ }^{41}$ Another U14 study found median peak linear accelerations, rotational velocities, and rotational accelerations of all game impacts (not just headers) to be $9.4 \mathrm{~g}, 4.1 \mathrm{rad} / \mathrm{s}$, and $689.1 \mathrm{rad} / \mathrm{s}^{2}$, respectively. ${ }^{33}$ While the level of play is different between youth, high school and university/collegiate female soccer players, it appears that exposure levels and peak kinematics are not significantly different but may depend more on coaching and playing style based on the limited data available.

We provided comprehensive exposure and peak impact kinematics metrics to allow for comparison and aggregation with data from prior studies. We did not find substantial differences between the head impact exposure per hour and exposure per athlete-exposure (AE). A previous American football study has reported large differences between these metrics due to variations in athlete participation during AEs. ${ }^{24}$ In our study, considering games/scrimmages and practices, the average exposure time per $\mathrm{AE}$ was close to one hour, as shown in Table 1 . It is likely that different sports will have different considerations in suitable exposure metrics to report. In the current study, we also reported both linear and angular head impact kinematics measured using a validated mouthpiece sensor. Furthermore, we computed both the peak angular velocity as well as peak change in angular velocity during impacts. Soccer heading impacts are active head impacts where the athlete directs their head towards the incoming ball, which leads to varying levels and directions of initial head velocity (Fig. 2). Due to initial head velocities, we found lower peak changes in angular velocity compared with peak velocity (Table 3). It is unclear what the effects of such initial velocities might be on brain injury risk, and future research may further investigate this effect to identify the most appropriate angular velocity metric to report.

In the current study, we have examined head exposure and biomechanics over a unique pandemic-affected soccer season, where most of the data came from practices. In addition, we did not have sensor data from every participant in all events during the season, due to practical limitations such as sensor malfunction and player injuries. Despite having over 1000 impacts for exposure and kinematics analysis across session type, player position, and contact scenario, certain categories had relatively fewer impacts (e.g. games and scrimmages, overhand/ground impacts, side impacts). As such, our exposure results may not be generalized to other teams or seasons. In addition, 206 video-confirmed impacts did not register on the mouthpiece sensors and the magnitude of these impacts are unknown. These impacts may have had lower magnitude than the $5 \mathrm{~g}$ trigger threshold, or may potentially be false negative detections due to other sensor error. The authors have also identified potential bias in triggering relatively lowmagnitude head impacts using the linear acceleration threshold method. ${ }^{52}$ A possible solution could be to lower the trigger sensor threshold below $5 \mathrm{~g}$ to capture potential low magnitude impacts.

In summary, our study has expanded on the current literature investigating on-field head impacts experienced by university women's soccer players. To our knowledge, this is the first study to thoroughly investigate head impact exposure and biomechanics across session type, player position, and contact scenarios in collegiate women's soccer using a mouthpiece sensor. Our findings on practice head impact exposure and kinematics as well as contributing factors to highkinematics head impacts could lead to improved monitoring and management of head impacts in soccer. Future research may involve larger scale recruitment over multiple seasons or combine data across studies to generate a larger dataset for impact exposure and kinematics analysis.

\section{ACKNOWLEDGMENTS}

The authors would like to thank UBC Women's Soccer team and the team coach Jesse Symons, whose support made this study possible. We thank Sandra 
Wong, Britney Ha, Ava Katz, Tyler Proctor, Annika Szarka, Ian Hunter and Joyce Kao for their invaluable help in field data collection and video analyses. Special thanks to the Wake Forest Center for Injury Biomechanics researchers Logan Miller, Konstantina Strates and Joel Stitzel for their collaboration and assistance in mouthpiece sensor deployment. We would also like to thank Susan Schmidt and Kaitlyn Spicer for assistance in dental molding. This study was supported by the Li Tze Fong Memorial Fellowship, the UBC FourYear Fellowship program, and the Canadian New Frontiers in Research Fund (Grant Number NFRFE2018-00141).

\section{CONFLICT OF INTEREST}

The authors report no conflicts of interest.

\section{REFERENCES}

${ }^{1}$ Babbs, C. F. Biomechanics of heading a soccer ball: implications for player safety. ScientificWorldJournal. 1:281-322, 2001. https://doi.org/10.1100/tsw.2001.56.

${ }^{2}$ Bartsch, A., E. Benzel, V. Miele, D. Morr, and V. Prakash. Impact 'fingerprints' and preliminary implications for an 'intelligent mouthguard' head impact dosimeter. Sport. Eng. 15:93-109, 2012.

${ }^{3}$ Bartsch, A., S. Samorezov, E. Benzel, V. Miele, and D. Brett, Validation of an 'intelligent mouthguard' single event head impact dosimeter, SAE Tech. Pap., vol. 201422-00, 2014, Accessed: Aug. 31, 2020. [Online]. https://w ww.sae.org/publications/technical-papers/content/2014-22$0001 /$.

${ }^{4}$ Beaudouin, F., et al. The UEFA Heading Study: heading incidence in children's and youth' football (soccer) in eight European countries. Scand. J. Med. Sci. Sports. 2020. h ttps://doi.org/10.1111/sms.13694.

${ }^{5}$ Beckwith, J. G., J. J. Chu, and R. M. Greenwald. Validation of a noninvasive system for measuring head acceleration for use during boxing competition. J. Appl. Biomech. 23(3):238-244, 2007. https://doi.org/10.1123/jab. 23.3.238.

${ }^{6}$ Caccese, J. B., L. C. Lamond, T. A. Buckley, and T. W. Kaminski. Reducing purposeful headers from goal kicks and punts may reduce cumulative exposure to head acceleration. Res. Sport. Med. 24(4):407-415, 2016. https://doi. org/10.1080/15438627.2016.1230549.

${ }^{7}$ Camarillo, D. B., P. B. Shull, J. Mattson, R. Shultz, and D. Garza. An instrumented mouthguard for measuring linear and angular head impact kinematics in american football. Ann. Biomed. Eng. 41(9):1939-1949, 2013. https://doi.org/ 10.1007/s10439-013-0801-y.

${ }^{8}$ Campbell, K. R., et al. Laboratory evaluation of the gForce Tracker ${ }^{\mathrm{TM}}$, a head impact kinematic measuring device for use in football helmets. Ann. Biomed. Eng. 44(4):1246-1256, 2016. https://doi.org/10.1007/s10439-0151391-7.

${ }^{9}$ Cobb, B. R., A. M. Tyson, and S. Rowson. Head acceleration measurement techniques: reliability of angular rate sensor data in helmeted impact testing. Proc. Inst. Mech. Eng. 232(2):176-181, 2017

${ }^{10}$ FIFA, 2018 FIFA World Cup ${ }^{\text {TM }}$ - Media Release Russia 2018, 2018. Accessed: May 25, 2021. [Online]. https://ww w.fifa.com/worldcup/news/more-than-half-the-world-watc hed-record-breaking-2018-world-cup.

${ }^{11}$ Filben, T. M., et al. Comparison of women's collegiate soccer header kinematics by play state, intent, and outcome. J. Biomech. 2021. https://doi.org/10.1016/J.JBIO MECH.2021.110619.

${ }^{12}$ Gabler, L. F., et al. On-field performance of an instrumented mouthguard for detecting head impacts in American football. Ann. Biomed. Eng. 48(11):2599-2612, 2020. h ttps://doi.org/10.1007/s10439-020-02654-2.

${ }^{13}$ Hanlon, E. M., and C. A. Bir. Real-time head acceleration measurement in girls' youth soccer. Med. Sci. Sports Exerc. 44(6):1102-1108, 2012. https://doi.org/10.1249/MSS.0b013 e3182444d7d.

${ }^{14}$ Harriss, A., A. M. Johnson, D. M. Walton, and J. P. Dickey. The number of purposeful headers female youth soccer players experience during games depends on player age but not player position. Sci. Med. Footb. 3(2):109-114, 2019. https://doi.org/10.1080/24733938.2018.1506591.

${ }^{15}$ Harriss, A., D. M. Walton, and J. P. Dickey. Direct player observation is needed to accurately quantify heading frequency in youth soccer. Res. Sport. Med. 26(2):191-198, 2018. https://doi.org/10.1080/15438627.2018.1431534.

${ }^{16}$ Hernandez, F., et al. Six degree-of-freedom measurements of human mild traumatic brain injury. Ann. Biomed. Eng. 43(8):1918-1934, 2015. https://doi.org/10.1007/s10439-0141212-4.

${ }^{17}$ Higgins, M., P. D. Halstead, L. Snyder-Mackler, and D. Barlow. Measurement of impact acceleration: mouthpiece accelerometer versus helmet accelerometer (Journal of Athletic Training (2007) 42, 1, (5-10)). J. Athl. Train. 43(1):109, 2008

${ }^{18}$ Holbourn, A. H. S. Mechanics of head injuries. Lancet. 242(6267):438-441, 1943.

${ }^{19}$ Jadischke, R., D. C. Viano, N. Dau, A. I. King, and J. McCarthy. On the accuracy of the head impact telemetry (hit) system used in football helmets. J. Biomech. 46(13):2310-2315, 2013. https://doi.org/10.1016/j.jbio mech.2013.05.030.

${ }^{20}$ King, D., P. Hume, M. Brughelli, and C. Gissane. Instrumented mouthguard acceleration analyses for head impacts in amateur rugby union players over a season of matches. Am. J. Sports Med. 43(3):614-624, 2015.

${ }^{21}$ Kirkendall, D. T., S. E. Jordan, and W. E. Garrett. Heading and head injuries in soccer. Sport. Med. 31(5):369386, 2001. https://doi.org/10.2165/00007256-200131050-00 006.

${ }^{22}$ Kontos, A. P., A. Dolese, R. J. Elbin, T. Covassin, and B. L. Warren. Relationship of soccer heading to computerized neurocognitive performance and symptoms among female and male youth soccer players. Brain Inj. 25(12):1234-1241, 2011. https://doi.org/10.3109/02699052.2011.608209.

${ }^{23}$ Kunz, M. "FIFA Big Count 2006," 2007. Accessed: Mar. 18, 2018. [Online]. http://www.fifa.com/mm/document/fifa facts/bcoffsurv/emaga 9384 10704.pdf.

${ }^{24}$ Kuo, C., L. Wu, J. Loza, D. Senif, S. C. Anderson, and D. B. Camarillo. Comparison of video-based and sensor-based head impact exposure. PLoS ONE. 13(6):e199238, 2018. h ttps://doi.org/10.1371/journal.pone.0199238.

${ }^{25}$ Lamond, L. C., J. B. Caccese, T. A. Buckley, J. Glutting, and T. W. Kaminski. Linear acceleration in direct head 
contact across impact type, player position, and playing scenario in collegiate women's soccer players. J. Athl. Train. 53(2):115-121, 2018. https://doi.org/10.4085/1062-6 050-90-17.

${ }^{26}$ Levendusky, T., C. Armstrong, et al. Impact characteristics of two types of soccer balls. Sci. Footb. (Routledge Revivals). 2021:407-415, 1988.

${ }^{27}$ Levitch, C. F., et al. Recent and long-term soccer heading exposure is differentially associated with neuropsychological function in amateur players. J. Int. Neuropsychol. Soc. 24(2):147-155, 2018. https://doi.org/10.1017/S1355617717 000790 .

${ }^{28}$ Liu, Y., et al. Validation and comparison of instrumented mouthguards for measuring head kinematics and assessing brain deformation in football impacts. Ann. Biomed. Eng. 48(11):2580-2598, 2020. https://doi.org/10.1007/s10439-02 0-02629-3.

${ }^{29}$ Lynall, R. C., et al. Head impact biomechanics in women's college soccer. Med. Sci. Sports Exerc. 48(9):1772-1778, 2016. https://doi.org/10.1249/MSS.0000000000000951.

${ }^{30}$ Margulies, S. S., and L. E. Thibault. A proposed tolerance criterion for diffuse axonal injury in man. J. Biomech. 25(8):917-923, 1992. https://doi.org/10.1016/0021-9290(92 )90231-O.

${ }^{31}$ McCunn, R., F. Beaudouin, K. Stewart, T. Meyer, and J. MacLean. Heading in football: incidence, biomechanical characteristics and the association with acute cognitive function - a three-part systematic review. Sport. Med. 2021. https://doi.org/10.1007/S40279-021-01492-Z.

${ }^{32}$ Meaney, D. F., and D. H. Smith. Biomechanics of concussion. Clin. Sports Med. 30(1):19-31, 2011. https://doi. org/10.1016/J.CSM.2010.08.009.

${ }^{33}$ Miller, L. E., et al. Characterizing head impact exposure in youth female soccer with a custom-instrumented mouthpiece. Res. Sport. Med. 28(1):55-71, 2020. https://doi.org/ 10.1080/15438627.2019.1590833.

${ }^{34}$ Miller, L. E., C. Kuo, L. C. Wu, J. E. Urban, D. B. Camarillo, and J. D. Stitzel. Validation of a custom instrumented retainer form factor for measuring linear and angular head impact kinematics. J. Biomech. Eng. 140(5):16, 2018. https://doi.org/10.1115/1.4039165.

${ }^{35}$ Naunheim, R. S., et al. Does soccer headgear attenuate the impact when heading a soccer ball? Acad. Emerg. Med. 10(1):85-90, 2003.

${ }^{36}$ Nevins, D., L. Smith, and J. Kensrud. Laboratory evaluation of wireless head impact sensor. Asia-Pacific Congr. Sport. Technol. 112:175-179, 2015. https://doi.org/10.1016/ j.proeng.2015.07.195.

${ }^{37}$ Ommaya, A. K., and T. A. Gennarelli. Cerebral concussion and traumatic unconsciousness. Correlation of experimental and clinical observations of blunt head injuries. Brain. 97(4):633-654, 1974.

${ }^{38}$ Patton, D. A., et al. Head impact sensor studies in sports: a systematic review of exposure confirmation methods. Ann. Biomed. Eng. 48(11):2497-2507, 2020. https://doi.org/10.1 007/s10439-020-02642-6.

${ }^{39}$ Patton, D. A., C. M. Huber, C. C. McDonald, S. S. Margulies, C. L. Master, and K. B. Arbogast. Video confirmation of head impact sensor data from high school soccer players. Am. J. Sports Med. 48(5):1246-1253, 2020. https://doi.org/10.1177/0363546520906406.

${ }^{40}$ Press, J., and S. Rowson. Quantifying head impact exposure in collegiate women's soccer. Clin. J. Sport Med. 27(2):104-110, 2017.
${ }^{41}$ Rich, A. M., et al. Development, validation and pilot field deployment of a custom mouthpiece for head impact measurement. Ann. Biomed. Eng. 47(10):2109-2121, 2019. h ttps://doi.org/10.1007/s10439-019-02313-1.

${ }^{42}$ Rowson, S., et al. Rotational head kinematics in football impacts: an injury risk function for concussion. Ann. Biomed. Eng. 40(1):1-13, 2012. https://doi.org/10.1007/s10 439-011-0392-4.

${ }^{43}$ Rowson, S., et al. Can helmet design reduce the risk of concussion in football? J. Neurosurg. 120:919-922, 2014. h ttps://doi.org/10.3171/2014.1.JNS13916.

${ }^{44}$ Salinas, C. M., F. M. Webbe, and T. T. Devore. The epidemiology of soccer heading in competitive youth players. J. Clin. Sport Psychol. 3(1):15-33, 2009. https://doi.org/10. $1123 /$ jcsp.3.1.15.

${ }^{45}$ Saunders, T. D., R. K. Le, K. M. Breedlove, D. A. Bradney, and T. G. Bowman. Sex differences in mechanisms of head impacts in collegiate soccer athletes. Clin. Biomech. 74:14-20, 2020. https://doi.org/10.1016/J.CLINBIO MECH.2020.02.003.

${ }^{46}$ Sharratt, A. U.K. lags Canada in ban on heading the ball," Healthing.ca, 2020. https://www.healthing.ca/wellness/agin $\mathrm{g} /$ brain-health/u-k-lags-canada-in-ban-on-heading-the-ball (accessed Aug. 23, 2021).

${ }^{47}$ Siegmund, G. P., K. M. Guskiewicz, S. W. Marshall, A. L. DeMarco, and S. J. Bonin. Laboratory validation of two wearable sensor systems for measuring head impact severity in football players. Ann. Biomed. Eng. 44(4):1257-1274, 2015. https://doi.org/10.1007/s10439-015-1420-6.

${ }^{48}$ Spiotta, A. M., A. J. Bartsch, and E. C. Benzel. Heading in soccer. Neurosurgery. 70(1):1-11, 2012. https://doi.org/10. 1227/NEU.0b013e31823021b2.

${ }^{49}$ Tomblin, B. T., et al. Characterization of on-field head impact exposure in youth soccer. J. Appl. Biomech. 37(1):36-42, 2021. https://doi.org/10.1123/JAB.2020-0071.

${ }^{50}$ Tyson, A. M., S. M. Duma, and S. Rowson. Laboratory evaluation of low-cost wearable sensors for measuring head impacts in sports. J. Appl. Biomech. 34(4):320-326, 2018. h ttps://doi.org/10.1123/JAB.2017-0256.

${ }^{51}$ U.S. Soccer Federation, "Concussions and Head Injuries," 2018. https://usclubsoccer.org/head-injuries/ (accessed Aug. 23, 2021).

${ }^{52}$ Wang, T., R. Kenny, and L. C. Wu. Head impact sensor triggering bias introduced by linear acceleration thresholding. Ann. Biomed. Eng. 49:3189-3199, 2021.

${ }^{53}$ Witol, A. D., and F. M. Webbe. Soccer heading frequency predicts neuropsychological deficits. Arch. Clin. Neuropsychol. 18(4):397-417, 2003. https://doi.org/10.1016/S0887-6 177(02)00151-8.

${ }^{54} \mathrm{Wu}, \mathrm{L}$. C., et al. In vivo evaluation of wearable head impact sensors. Ann. Biomed. Eng. 44(4):1234-1245, 2015.

${ }^{55} \mathrm{Wu}, \mathrm{L}$. C., et al. In vivo evaluation of wearable head impact sensors. Ann. Biomed. Eng. 44(4):1234-1245, 2016. h ttps://doi.org/10.1007/s10439-015-1423-3.

${ }^{56} \mathrm{Wu}, \mathrm{L}$. C., et al. Bandwidth and sample rate requirements for wearable head impact sensors. J. Biomech. 49(13):29182924, 2016. https://doi.org/10.1016/j.jbiomech.2016.07.004.

Publisher's Note Springer Nature remains neutral with regard to jurisdictional claims in published maps and institutional affiliations. 